\title{
Construction of special eye models for investigation of chromatic and higher-order aberrations of eyes
}

\author{
Yi Zhai ${ }^{a, b}$, Yan Wang ${ }^{c,}$, Zhaoqi Wang ${ }^{\mathrm{a}, \mathrm{b}}$, Yongji Liu ${ }^{\mathrm{a}, \mathrm{b}}$, Lin Zhang ${ }^{\mathrm{c}}$, Yuanqing $\mathrm{He}^{\mathrm{a}, \mathrm{b}}$ and \\ Shengjiang Chang ${ }^{\mathrm{a}, \mathrm{b}}$ \\ ${ }^{a}$ Institute of Modern Optics, Nankai University, Tianjin 300071, China \\ ${ }^{b}$ Key Laboratory of Optical Information Science and Technology, Ministry of Education, Tianjin \\ 300071, China \\ ${ }^{c}$ Tianjin Eye Hospital \& Eye Institute, Tianjin Key Lab of Ophthalmology and Visual Science, Tianjin \\ Medical University, Tianjin 300020, China
}

\begin{abstract}
An achromatic element eliminating only longitudinal chromatic aberration (LCA) while maintaining transverse chromatic aberration (TCA) is established for the eye model, which involves the angle formed by the visual and optical axis. To investigate the impacts of higher-order aberrations on vision, the actual data of higher-order aberrations of human eyes with three typical levels are introduced into the eye model along visual axis. Moreover, three kinds of individual eye models are established to investigate the impacts of higher-order aberrations, chromatic aberration (LCA+TCA), LCA and TCA on vision under the photopic condition, respectively. Results show that for most human eyes, the impact of chromatic aberration on vision is much stronger than that of higher-order aberrations, and the impact of LCA in chromatic aberration dominates. The impact of TCA is approximately equal to that of normal level higher-order aberrations and it can be ignored when LCA exists.
\end{abstract}

Keywords: Visual optics, eye model, optical design, optical effects on vision, aberrations

\section{Introduction}

It is well known that the human eyeball as an optical system suffers from higher-order monochromatic and chromatic aberrations. With the use of psychophysical experiments, point-spread functions (PSFs), and modulation transfer functions (MTFs), the deteriorate effect of higher-order aberrations on visual quality can be revealed [1-3]. As a result, corrections of higher-order aberrations have to be applied to increase visual quality [4,5]. Clinical studies have shown that the longitudinal chromatic aberration (LCA) is about $2 \mathrm{D}$ across the visible spectrum without significant change among different individuals [6,7]. As for the transverse chromatic aberration (TCA), it is subject to high individual dif-

\footnotetext{
${ }^{*}$ Corresponding author: Yan Wang, Tianjin Eye Hospital \& Eye Institute, Tianjin Key Lab of Ophthalmology and Visual Science, Tianjin Medical University, No. 4, Gansu Road, Heping District, Tianjin 300020, China. Tel.: +86 22 27305083; Fax: +86 2227313336 82068; E-mail: wangyan7143@vip.sina.com.
}

0959-2989/14/\$27.50 @ 2014 - IOS Press and the authors. 
ferences with a typical value of approximately 0.6 arcmin [8-10]. Moreover, many studies have been con ducted to examine the effect of chromatic aberration on visual quality and to investigate the visual benefit of correcting higher-order and chromatic aberrations in contrast sensitivity and visual acuity [11-13].

The impacts of higher-order and chromatic aberrations on vision can be investigated with the help of appropriate eye models [14,15]. Zhang et al. [16] and He et al. [17] developed two individual eye models, without and with the angle formed by visual and optical axis. The first eye model considers LCA only, and the second eye model considers both LCA and TCA. However, with these models, we cannot directly obtain the TCA-only impact on vision.

In this paper, we firstly propose a novel eye model containing an achromatic element that excludes LCA while maintains TCA. As a result, it can be used to investigate the TCA-only impact on vision. Introducing the actual data for human eye's higher-order aberrations into eye model along the visual axis, three individual eye models are established to investigate the impacts of higher-order aberrations, chromatic aberrations (LCA+TCA), LCA and TCA on vision under the photopic condition, respectively.

\section{Methods}

\subsection{Construction of three individual eye models}

\subsubsection{The first eye model}

With the help of optical design software Zemax, the eye model with visual axis accordant to optical axis is established, upon which eyeball visual performance under the photopic condition with LCAonly can be investigated. Employing the improved Gullstrand-Le Grand eye model [17] as the initial

Table 1

Structural parameters of the first eye model

\begin{tabular}{llllll}
\hline Surface & Refractive Index & Abbe Number & Radius $(\mathrm{mm})$ & Semi-diameter $(\mathrm{mm})$ & Thickness $(\mathrm{mm})$ \\
\hline Anterior Cornea & 1.38 & 55.80 & 7.80 & 1.69 & 0.55 \\
Posterior Cornea & 1.34 & 52.80 & 6.50 & 1.67 & 3.05 \\
Anterior Crystalline Lens & 1.42 & 49.80 & 10.20 & 1.49 & 4.00 \\
Posterior Crystalline Lens & 1.34 & 52.80 & -6.00 & 1.27 & 16.55 \\
Retina & & -12.50 & 6.00 & \\
\hline
\end{tabular}

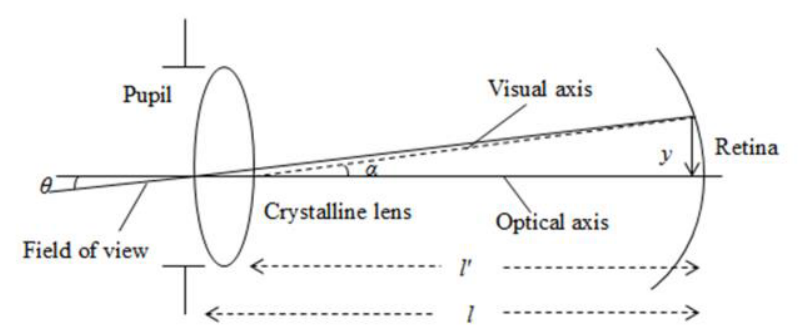

Fig. 1. Layout showing the relationship between $\alpha$ and $\theta$. 
structure, we set the pupil diameter and the primary wavelength to $3 \mathrm{~mm}$ and $555 \mathrm{~nm}$, respectively. Optimizing the conic of the posterior surface of crystalline lens and the thickness of vitreous, the MTF could approach the diffraction limit. The structural parameters of the first kind of eye model are given in Table 1. Five wavelengths, including $470 \mathrm{~nm}, 510 \mathrm{~nm}, 555 \mathrm{~nm}, 610 \mathrm{~nm}$ and $650 \mathrm{~nm}$, are used and weighted by the luminosity function curve under the photopic condition. And LCA is about 1.33 D.

\subsubsection{The second eye model}

To investigate the impact of chromatic aberration (LCA+TCA) on vision, we establish the second eye model which involves the angle formed by visual and optical axis, based on the first eye model. The visual axis intersects the optical axis at the nodal point in the vicinity of posterior surface of crystalline lens, with a typical value of $\alpha$ of $5^{\circ}$ in the temporal side and $1.5^{\circ}$ in the down side [18]. The angle $\alpha$ can be transferred into the field of view (FOV), $\theta$, as shown in Figure 1. The relation between the height of the retina image formed by the ray along visual axis and that formed by the chief ray of $\theta$ is given by

$$
y=l \cdot \tan \theta=l^{\prime} \cdot \tan \alpha
$$

where $y$ is the image height on retina, $l / l^{\prime}$ is the distance between the anterior/posterior surface of crystalline lens and the retina. Substituting the typical value of $\alpha$ into Eq. (1), the value of $\theta$ is $4^{\circ}$ in the temporal side (y-direction) and $1.2^{\circ}$ in the down side (x-direction). Add the angle values of FOV to the first eye model and then perform optimization as follows:

- Set the type of the anterior corneal surface to "Zernike Fringe Sag" with 4 15 Zernike coefficients as variables and carry out optimization;

- Set the type of the posterior surface of crystalline lens to "Even Asphere" with 4th-order, 6thorder and 8th-order polynomial coefficients as variables and complete optimization.

The MTFs at FOV of zero and $\theta$ degree are both close to the diffraction limit after optimization. As shown in Figure 2(a), the optical axis (blue line) is along the direction of FOV of zero degree, and the visual axis (green line) is along the direction of FOV of $1.2^{\circ}$ in $\mathrm{x}$-direction and $4^{\circ}$ in y-direction. Finally, the establishment is completed by rotating the entire eye model with the center of posterior surface of crystalline lens as the rotation center to make the visual axis in the direction of FOV of zero degree and the optical axis in the direction of FOV of $-1.2^{\circ}$ in x-direction and $-4^{\circ}$ in y-direction, as shown in Figure 2(b). For the second eye model, LCA is about $1.33 \mathrm{D}$ and TCA is approximately $4.94 \mu \mathrm{m}$ in the wavelength range from 470 to $650 \mathrm{~nm}$.

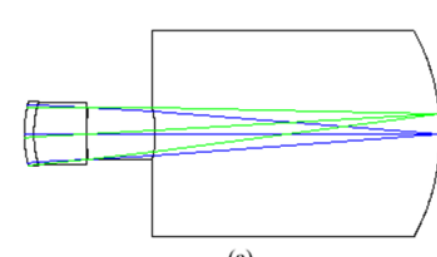

(a)

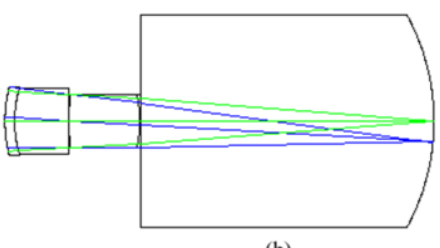

(b)

Fig. 2. Layout of the second eye model. (a) Before rotation and (b) After rotation. 


\subsubsection{The third eye model}

To investigate the TCA-only impact on vision, the third eye model which eliminates LCA is established by inserting an achromatic element into the second eye model as shown in Figure 2(a). Generally, an achromatic element consists of a concave and a convex lens with materials of different dispersions, the cemented doublet. As the achromatic element introduces additional monochromatic aberrations, the correction of the aberrations needs to be taken into account. Diffractive optical elements have excellent achromatic performances, and a refractive-diffractive hybrid system would not only correct chromatic aberration but also introduce a smaller amount of monochromatic aberrations [19]. In Zemax, the diffractive surface is described using the Binary type with a phase function given by

$$
\Phi=\sum_{i=1}^{N} A_{i} \rho^{2 i}
$$

where $A_{i}$ is the coefficient on the $2 i^{\text {th }}$ power of $\rho$ with $\rho$ being the normalized radial coordinate of aperture. $N$ is the number of polynomial coefficients in the series. The second-order phase coefficient of $A_{1}$ is for the correction of chromatic aberration. To separately analyze the impact of TCA on vision, the achromatic element needs to be designed to correct only LCA while maintain TCA. From the optical design perspective, the performance of an achromatic element largely depends on its location in the optical system $[19,20]$. Therefore, three types of refractive-diffractive hybrid achromatic elements for eliminating LCA are designed with three typical locations used, including location in front of the cornea, in front of the pupil and behind the crystalline lens.

1. In front of the cornea

Sequentially insert a convex and a concave lens in front of the cornea in the second eye model, with the distance from the posterior surface of concave lens to the anterior corneal surface to be $0.1 \mathrm{~mm}$, and then perform a two-step optimization as follows:

- Introduce the operand of effective focal length "EEFL" into the merit function and then optimize the curvature radii of each surface of two lenses;

- Set the posterior surface of concave lens as a binary surface, and introduce the operand "AXCL" into the merit function for LCA correction. Then optimize the second-order phase coefficient of $\mathrm{A} 1$ and the curvature radius of binary surface.

After optimization, the residual LCA is only $0.03 \mathrm{D}$, yet the TCA increases from 4.94 to $8.95 \mu \mathrm{m}$.

2. Behind the crystalline lens

Sequentially insert the convex and concave lens behind the crystalline lens in the second eye model, with the distance from the anterior surface of convex lens to the posterior surface of crystalline lens to be $0.1 \mathrm{~mm}$, and then perform the above optimization steps. As a consequence, the residual LCA is just $0.004 \mathrm{D}$, while the TCA decreases from 4.94 to $1.46 \mu \mathrm{m}$.

3. In front of the pupil

Sequentially insert the convex and concave lens into the anterior chamber in the second eye model, with its location near to the pupil, and then perform the above optimization steps. Adjust the thickness and location of two lenses to make sure that LCA is completely eliminated while TCA is maintained. After optimization, the residual LCA is only $0.03 \mathrm{D}$ and TCA only slightly changes to $4.99 \mu \mathrm{m}$. The layout of the LCA corrector located in front of the pupil is shown in Figure 3 with its structure parameters given in Table 2. Based on the eye model shown in Figure 3, we continue to establish the third eye model to investigate the TCA-only impact with the following steps: 
Table 2

Structural parameters of the LCA corrector located in front of the pupil

\begin{tabular}{lllll}
\hline & & Radius $(\mathrm{mm})$ & Thickness $(\mathrm{mm})$ & Glass \\
\hline \multirow{2}{*}{ Convex Lens } & Anterior surface & 44.78 & 0.32 & \multirow{2}{*}{ BK6 } \\
& Posterior surface & -7.33 & 0.26 & \\
\multirow{2}{*}{ Concave Lens } & Anterior surface & -7.97 & 0.10 & \multirow{2}{*}{ F8 } \\
& Posterior surface & 671.91 & & \\
\hline
\end{tabular}

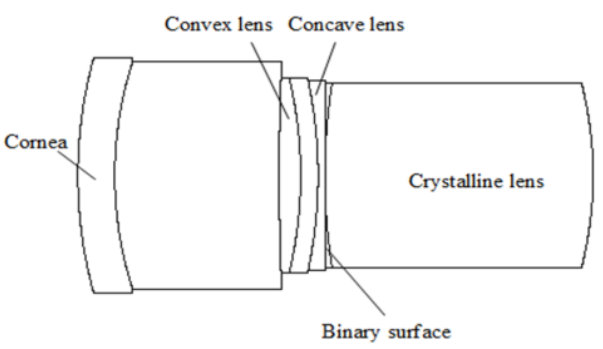

Fig. 3. LCA corrector located in front of the pupil.

- Optimize the shapes of the anterior corneal surface and the posterior surface of crystalline lens again to correct additional monochromatic aberrations introduced by the LCA corrector. As a result, the MTFs at the primary wavelength of $555 \mathrm{~nm}$ are approaching the diffraction limit at FOV with both of zero and $\theta$ degree;

- Complete the establishment of the eye model for TCA-only impacts by rotating the eye model with LCA corrector to ensure that the visual axis is in the direction of FOV of zero degree and the optical axis is in the direction of FOV of $-1.2^{\circ}$ in $\mathrm{x}$-direction and $-4^{\circ}$ in $\mathrm{y}$-direction.

\subsection{Measurement and introduction of higher-order aberrations}

A group including 127 eyes of 79 subjects was selected and among them 96 eyes are from 48 subjects. All eyes are without ocular disease or corneal surgery. Wavefront aberrations are measured with Hartmann-Shack wavefront aberrometer, the Wavescan, in a darkroom with natural pupil diameter ranging from 5.5 to $7.5 \mathrm{~mm}$ in the Tianjin Eye Hospital. The Wavescan can measure spherical refractive errors between $-12.0 \mathrm{D}$ and $+9.0 \mathrm{D}$, cylindrical refractive errors up to $5.0 \mathrm{D}$, with the accuracy of both of $0.1 \mathrm{D}$. The measurement of higher-order aberrations is with Zernike polynomials of seventhorder (35 items) [21]. The working wavelength is $780 \mathrm{~nm}$ and the measuring time is $0.2 \mathrm{~s}$. The study protocol was approved by Tianjin Eye Hospital Institutional Review Board and all participants provided written informed consent.

A conversion of wavefront aberrations from the actual pupil diameter to a fixed pupil diameter of 3 $\mathrm{mm}$ performed using Matlab [22]. The value of the root-mean-square of higher-order aberrations $\left(\mathrm{RMS}_{\mathrm{H}}\right.$, third-order to sixth-order Zernike polynomials) of 127 eyes ranges from 0.009 to $0.097 \mu \mathrm{m}$. Table 3 gives the statistical distribution of $\mathrm{RMS}_{\mathrm{H}}$ values of eyes with $3 \mathrm{~mm}$ pupil. As we can see, there are 3 eyes with the value less than $0.01 \mu \mathrm{m}$ and 1 eye with the value greater than $0.09 \mu \mathrm{m}$. It can also be seen that there are 110 eyes with the value less than $0.04 \mu \mathrm{m}$, which account for $86.6 \%$ of the total number of eyes. 
Table 3

Statistical distribution of RMSH values of 127 eyes

\begin{tabular}{lllllll}
\hline $\mathrm{RMS}_{\mathrm{H}}(\mu \mathrm{m})$ & $<0.01$ & $0.01 \sim 0.019$ & $0.02 \sim 0.029$ & $0.03 \sim 0.039$ & $0.04 \sim 0.049$ & $>0.09$ \\
\hline Number of eyes & 3 & 35 & 40 & 32 & 16 & 1 \\
\hline
\end{tabular}

According to the distribution of $\mathrm{RMS}_{\mathrm{H}}$ values, we define eyes with $\mathrm{RMS}_{\mathrm{H}}$ of $0.01,0.04$ and $0.09 \mu \mathrm{m}$ as higher-order aberrations with minimum level, normal level and high level, respectively. This definition is used to evaluate the impacts of higher-order aberrations on vision. To introduce higher-order aberrations into the three eye models along visual axis, a phase plane denoted as the Zernike Fringe Phase type with Zernike coefficients corresponding to the actual values of higher-order aberrations is inserted in front of the anterior corneal surface in the models.

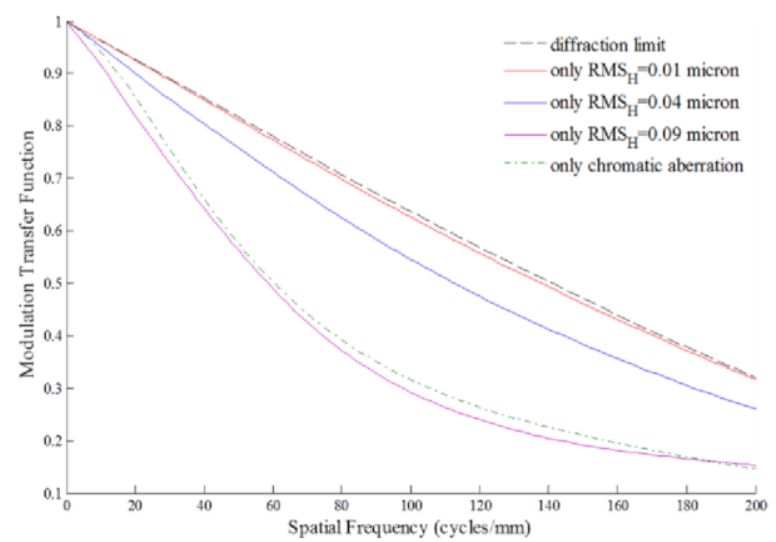

Fig. 4. The radial averaged MTFs with only higher-order aberrations (solid lines), only chromatic aberration (green dashed-dotted line) and the diffraction limit (dashed line). The curves of red, blue and purple solid lines represent the MTF with higher-order aberrations with RMS values of $0.01,0.04$ and $0.09 \mu \mathrm{m}$, respectively.

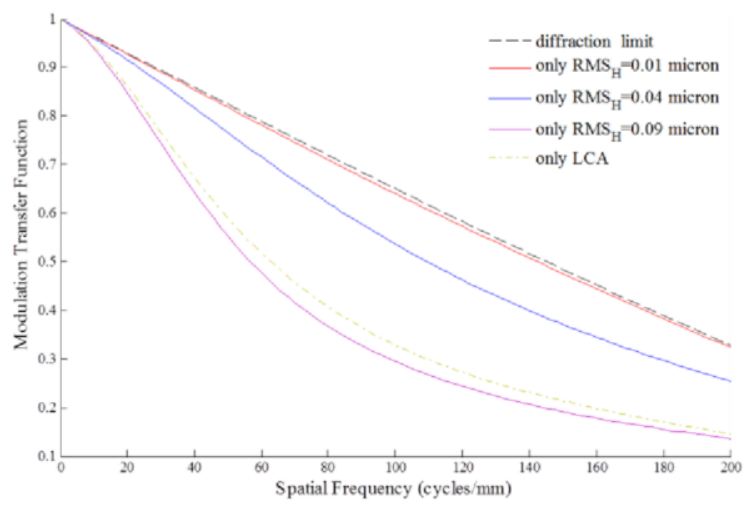

Fig. 5. The radial averaged MTFs with only higher-order aberrations (solid lines), only LCA (dark yellow dashed-dotted line) and the diffraction limit (dashed line). The curves of red, blue and purple solid lines represent the MTF with higherorder aberrations with RMS values of $0.01,0.04$ and $0.09 \mu \mathrm{m}$, respectively. 


\section{Results and discussions}

In the second eye model, the effect of chromatic aberration (LCA+TCA) on vision is evaluated by the polychromatic MTF, and the diffraction limit is represented by the MTF at the primary wavelength of $555 \mathrm{~nm}$. Three typical levels of higher-order aberrations are introduced into the model with their impacts on vision being characterized by the monochromatic MTFs. The results are shown in Figure 4, where the solid lines denote the radial averaged MTFs with only higher-order aberrations with RMS values of $0.01,0.04$ and $0.09 \mu \mathrm{m}$, respectively. The dashed-dotted line denotes the radial averaged MTF with only chromatic aberration, and the dashed line denotes the MTF with diffraction limit. It can be seen that the chromatic aberration seriously attenuates the MTF. Besides, the MTF gradually decreases when higher-order aberration increases. When higher-order aberration increases to $0.09 \mu \mathrm{m}$ of RMS value, the attenuation of MTF is similar to that with only chromatic aberration. However, in the statistics of human eyes in this paper, there is only one eye with a $\mathrm{RMS}_{\mathrm{H}}$ value over $0.09 \mu \mathrm{m}$, and most eyes are with $\mathrm{RMS}_{\mathrm{H}}$ values below $0.04 \mu \mathrm{m}$. It can be seen from Figure 4 that the attenuation of MTF by normal level of higher-order aberration with RMS value of $0.04 \mu \mathrm{m}$ is much less than that by only chromatic aberration.

With the first eye model, the impacts of LCA and higher-order aberrations on vision are separately investigated and the results are shown in Figure 5. The radial averaged MTFs with only higher-order aberrations of three typical levels are denoted by solid lines and the polychromatic radial averaged MTF with LCA-only is denoted by the dashed-dotted line. The dashed line represents the MTF with diffraction limit. It can be seen from Figure 5 that, in comparison with MTF of only higher-order aberrations, the attenuation imposed by LCA is much stronger than that by normal level higher-order aberrations $\left(\mathrm{RMS}_{\mathrm{H}}=0.04 \mu \mathrm{m}\right)$ and is approximately equal to that by high level higher-order aberrations $\left(\mathrm{RMS}_{\mathrm{H}}=0.09 \mu \mathrm{m}\right)$. Comparing the dashed-dotted lines in Figures 4 and 5, it can be seen that the MTF with LCA-only (dark yellow line) is quite similar to that with only LCA+TCA (green line), indicating that the influence of TCA can be ignored in the presence of LCA.

The impacts of TCA and higher-order aberrations on vision are evaluated with the third eye model and the results are shown in Figure 6, where the dashed-dotted line represent the polychromatic radial averaged MTF with TCA-only, the solid lines represent the radial averaged MTFs with only higherorder aberrations of three typical levels and the dashed line represents the MTF with diffraction limit. In comparison with MTF of only higher-order aberrations, it can be seen that the impact of TCA is approximately equal to the impact of normal level of higher-order aberrations. Comparing the radial averaged MTF with LCA-only denoted by the dashed-dotted line in Figure 5, the impact of TCA is much smaller, especially in the medium and low spatial frequencies, where the important spatial frequencies of vision locate.

\section{Conclusion}

With the help of optical design software Zemax, we demonstrate that the achromatic element should be placed just in front of the pupil of eye model to eliminate LCA while maintain TCA. Three individual eye models that higher-order aberrations can be introduced along the visual axis are established. The first model is with visual axis accordant to optical axis, the second involves the angle formed by visual and optical axis and the third includes an achromatic element to correct LCA only which exists in the second model. Furthermore, higher-order aberrations of human eyes are fed into the eye models along visual axis. The impacts of higher-order aberrations, chromatic aberration (LCA+TCA), LCA 


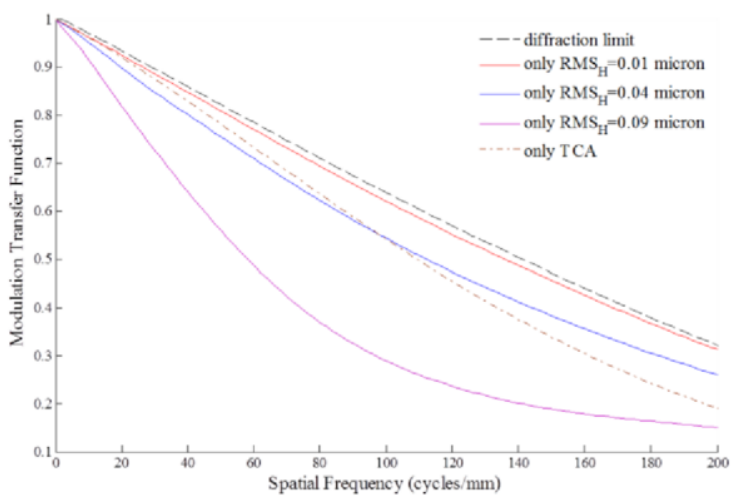

Fig. 6. The radial averaged MTFs with only higher-order aberrations (solid lines), only TCA (brown dashed-dotted line) and the diffraction limit (dashed line). The curves of red, blue and purple solid lines represent the MTF with higher-order aberrations with RMS values of $0.01,0.04$ and $0.09 \mu \mathrm{m}$, respectively.

and TCA on vision under the photopic condition are then investigated using MTFs based on the three models, respectively. Conclusions are as follows:

- When the achromatic element is placed in front of the cornea, the LCA is eliminated but the TCA increases. When the achromatic element is placed behind the crystalline lens, the LCA is eliminated but the TCA decreases. To eliminate LCA while maintain TCA, the achromatic element has to be placed in front of the pupil.

- For most human eyes, the impact of chromatic aberration (LCA+TCA) on vision under the photopic condition is much stronger than that of higher-order aberrations.

- The impact of LCA on vision is almost equal to that of LCA+TCA. In the presence of LCA, the impact of TCA on vision can be ignored.

- The impact of TCA is approximately equal to that of normal level higher-order aberrations and is much weaker than that of LCA on vision.

It should be emphasized that chromatic aberrations, especially LCA, have positive effects on the enhancement of depth of focus of human eyes $[2,23]$. This statement is out of the scope of this paper and will be reported in another paper.

\section{Acknowledgement}

This work is supported by the National Nature Science Foundation of China (No. 81 170873), and the National Nature Science Foundation of China (No. 11104149).

\section{References}

[1] A. Guirao, J. Porter, D.R. Williams and I.G. Cox, Calculated impact of higher-order monochromatic aberrations on retinal image quality in a population of human eyes, J. Opt. Soc. Am. A 19 (2002), 620-628.

[2] S. Ravikumar, L.N. Thibos and A. Bradley, Calculation of retinal image quality for polychromatic light, J. Opt. Soc. Am. A 25 (2008), 2395-2407.

[3] D.A. Atchison and H. Guo, Subjective blur limits for higher order aberrations, Optometry and Vision Science 87 (2010), 890-898. 
[4] G.Y. Yoon, I. Cox and D.R. Williams, The visual benefit of static correction of the monochromatic wave aberration, Invest. Ophthalmol. Visual Sci. 40 (1999), B171.

[5] G.Y. Yoon, T.M. Jeong, I.G. Cox and D.R. Williams, Vision improvement by correcting higher-order aberrations with phase plates in normal eyes, Journal of Refractive Surgery 20 (2004), 523-527.

[6] J.G. Sivak and T. Mandelman, Chromatic dispersion of the ocular media, Vision Res. 16 (1982), 997-1003.

[7] P.A. Howarth and A. Bradley, The longitudinal chromatic aberration of the human eye and its correction, Vision Res. 26 (1986), 361-366.

[8] Y.U. Ogboso and H.E. Bedell, Magnitude of lateral chromatic aberration across the retina of the human eye, J. Opt. Soc. Am. A 4 (1987), 1666-1672.

[9] P. Simonet and M.C. Campbell, The optical transverse chromatic aberration on the fovea of the human eye, Vision Res. 30 (1990), 187-206.

[10] L.N. Thibos, A. Bradley, D.L. Still, X. Zhang and P.A. Howarth, Theory and measurement of ocular chromatic aberration, Vision Res. 30 (1990), 33-49.

[11] G.Y. Yoon and D.R. Williams, Visual benefit of correcting the higher order monochromatic aberrations and the chromatic aberration in the eye, Vision Science and its Applications 35 (2000), 205-211.

[12] G.Y. Yoon and D.R. Williams, Visual performance after correcting the monochromatic and chromatic aberrations of the eye, J. Opt. Soc. Am. A 19 (2002), 266-275.

[13] F. Autrusseau, L.N. Thibos and S.K. Shevell, Chromatic and wavefront aberrations: L-, M-and S-cone stimulation with typical and extreme retinal image quality, Vision Res. 51 (2011), 2282-2294.

[14] K. Ohnuma, H. Kayanuma, T. Lawu, K. Negishi, T. Yamguchi and T. Noda, Retinal image contrast obtained by a model eye with combined correction of chromatic and spherical aberrations, Biomedical Optics Express 2 (2011), $1443-1451$.

[15] S. Giovanzana, H.T. Kasprzak, B. Palucki and Ş. Ţălu, Non-rotational aspherical models of the human optical system, Journal of Modern Optics 60 (2013), 1898-1904.

[16] M. Zhang, Z. Wang, Y. Wang and T. Zuo, Studies of transverse chromatic aberration based on individual eye model, Optik 121 (2010), 2080-2086.

[17] Y. He, Y. Wang, Z. Wang, C. Fang, Y. Liu, L. Zhang, S. Zheng, L. Wang and S. Chang, Study on chromatic aberration in a population of Chinese myopic eyes by means of optical design, Biomedical Optics Express 4 (2013), 667679.

[18] Y. Le Grand and S.G. El Hage, Physiological Optics, Springer Series in Optical Sciences, Springer, 1980.

[19] M. Zhang, Research on chromatic aberration of human eye based on individual eye, Ph.D. Dissertation, Nankai University, 2010.

[20] M. Zhang, Z. Wang, Y. Wang and T. Zuo, The study of correction for chromatic aberrations of human eye with diffractiverefractive hybrid elements, Optik 121 (2010), 2209-2212.

[21] F. Cade, A. Cruzat, E.I. Paschalis, L.E. Santo and R. Pineda, Analysis of four aberrometers for evaluating lower and higher order aberrations, Plos One 8 (2013), e54990.

[22] H. Guo, Z. Wang, Q. Zhao, W. Quan and Y. Wang, Individual eye model based on wavefront aberration, Optik 116 (2005), 80-85.

[23] F.W. Campbell, The depth of field of the human eye, Optica. Acta. 4 (1957), 157-164. 